\title{
Localization of Synaptotagmin in the Regenerating Sprouts Emanating from the Nodes of Ranvier
}

\author{
Kaori Uehara*, Akira Mizoguchi**, Kosaku Mizuno* and Chizuka Ide** \\ *Department of Orthopaedic Surgery, Kobe University School of Medicine, Kobe 650 and ${ }^{* *}$ Department of Anatomy, \\ Cell Biology and Neurobiology, Faculty of Medicine, Kyoto University, Kyoto, 606-01
}

Received for publication October 8, 1995

\begin{abstract}
Localization of synaptotagmin, a $\mathrm{Ca}^{2+}$-binding protein associated with synaptic vesicles, in regenerating axons was investigated by immunocytochemistry in the injured rat sciatic nerves. The early regenerating axonal sprouts emanating from nodes of Ranvier exhibited synaptotagmin immunoreactivity on vesicles, vacuoles and surface plasma membranes. In the welldeveloped regenerating sprouts extending through the space between Schwann cell basal lamina and myelin sheath of the parent axon, the growing tips, i.e., typical growth cones, exhibited an intense immunoreaction on vesicles, vacuoles and surface plasma membranes, while the stem regions where
\end{abstract}

the sprouts were continuous with the parent axon exhibited almost no immunoreaction on any organelles including plasma membranes. These findings suggest that synaptotagmin-immunoreactive vesicles and vacuoles might be utilized for the supply of membrane components to the surface plasma membrane in the growth cone. Synaptotagmin which is known to regulate membrane fusion of synaptic vesicles with presynaptic plasma membranes in a $\mathrm{Ca}^{2+}$. dependent manner in synapses may participate in the regulation of the membrane fusion between the vesicles and plasma membranes in growth cones.

Key words: Synaptotagmin (p65), Nerve regeneration, Growth cone, Plasmalemmal expansion, $\mathrm{Ca}^{2+}$-dependent vesicle fusion

\section{Introduction}

The initiation of peripheral nerve regeneration following injury is morphologically defined as the sprouts emanating from the nodes of Ranvier proximal to the lesion, which were called 'germinative zone' by Cajal $[6,10,11$, 20]. The node of Ranvier is a cleft between neighboring myelin sheaths, where the axon is covered by Schwann cell processes. The basal laminae of neighboring Schwann cells are continuous at the node, implying that each basal lamina tube contains one myelinated fiber $[2,3,12,24$, 27]. The nodal axolemma is characterized by the submembraneous dense coat. The earliest regenerating sprouts initiate as small evaginations of the nodal axolemma devoid of the submembraneous dense coat $[13,15,19,28]$. The small evaginations contain small vesicles (ca, $50 \mathrm{~nm}$ in diameter), vacuoles (100-200 nm) and multivesicular

Correspondence to: Dr. Akira Mizoguchi, Department of Anatomy, Cell Biology and Neurobiology, Faculty of Medicine, Kyoto University, Konoe-cho, Yoshida, Sakyo-ku, Kyoto 606-01, Japar. bodies. As the evaginations grow out as sprouts, they contact with basal lamina of Schwann cells and extend along the inner surface of the basal lamina $[13,14]$. The tips of these sprouts are filled with the vesicular organelles as described above and called growth cones, while the stem regions of the sprouts where they are continuous with the parent axons contain cytoskeletal elements such as neurofilaments and microtubules. These cytoskeletal elements are considered to regulate the mobility of the sprouts $[5,17,29]$.

During the axonal sprouting, a great deal of membrane is continuously required for the axolemmal expansion. The source of the membrane for the rapidly growing axolemma and the mode by which the membrane is added to the axolemma have been of primary interest. Pfeninger et al. have demonstrated in vitro that some clear vesicles localized in growth cones fuse with the axolemma in a $\mathrm{Ca}^{2+}$-dependent manner $[22,23]$. The molecules involved in the $\mathrm{Ca}^{2+}$-dependent membrane fusion in the growth cones are, however, largely unknown.

We have here immunohistochemically investigated the 
localization of synaptotagmin, which is regarded as the key molecule regulating the $\mathrm{Ca}^{2+}$-dependent neurotransmitter release in synapse [see for reviews, 7, 26]. Synaptotagmin is a synaptic vesicle-associated protein which was initially found as p 65 [18]. The analysis of the primary structure has revealed that synaptotagmin has a single membrane-spanning domain and two C-2 domains which bind phospholipids in a $\mathrm{Ca}^{2+}$-dependent manner just as the $\mathrm{C}-2$ domains of protein kinase $\mathrm{C}$, phospholipase A2 and Rabphilin-3A do. Recent studies have shown that $\mathrm{Ca}^{2+}$-dependent membrane fusion of synaptic vesicles with presynaptic plasma membranes is impaired in synaptotagmin knockout mice [8]. These findings indicate that synaptotagmin functions as a $\mathrm{Ca}^{2+}$-sensor to regulate exocytosis of synaptic vesicles in synapse.

In the present study, we have demonstrated that synaptotagmin is localized on the vesicles and plasma membranes of the early regenerating sprouts emanating from the nodes of Ranvier in injured rat sciatic nerve and that it is especially concentrated in the growth cones of well-developed regenerating sprouts.

\section{Materials and Methods}

\section{Injury of the nerve}

Adult Sprague-Dawley female rats weighing 200$250 \mathrm{~g}$ were used in the present study. The animals were anesthetized by intraperitoneal injection of Nembutal (sodium pentobarbiturate $50 \mathrm{mg} / \mathrm{kg}$ body weight), and the left sciatic nerve was exposed at the mid-thigh level. The nerve was kept ligated with 1-0 silk thread for $24 \mathrm{hr}$, and released from the ligation $24 \mathrm{hr}$ prior to sacrifice (ligation injury). By ligation, axons were injured, but the continuity of the endoneurial tubes, i.e. Schwann cell basal lamina tubes was preserved $[9,14]$.

\section{Immunocytochemistry}

Animals were anesthetized with ether and fixed by transcardiac perfusion with a fixative containing $2 \%$ paraformaldehyde in PBS $(0.15 \mathrm{M} \mathrm{NaCl}$ in $20 \mathrm{mM}$ sodium phosphate buffer at $\mathrm{pH} 7.5$ ) supplemented with $8 \%$ sucrose. The proximal segment was removed together with a distal segment in the ligation injury and immersed in the same fixative as above for $4 \mathrm{hr}$ at $4^{\circ} \mathrm{C}$. The specimens were cryoprotected through a range of increasing sucrose concentrations $(10,15,20$, and $25 \%)$ in PBS, embedded in OCT compound, quick-frozen, and sectioned $15 \mu \mathrm{m}$ thick in a cryostat. The frozen sections were mounted on slides, washed with $50 \mathrm{mM}$ Tris buffer for $30 \mathrm{~min}$ and then incubated with PBS containing 5\% bovine serum albumin (BSA) containing $0.005 \%$ saponin. These sections were incubated for $48 \mathrm{hr}$ with antisynaptotagmin monoclonal antibody (mAb) at a concentration of $5 \mu \mathrm{g} / \mathrm{ml}$ in PBS containing $0.005 \%$ saponin. The specificity of the anti-synaptotagmin $\mathrm{mAb}$, which was kindly supplied by Dr. Masami Takahashi (Mitsubishi Kasei Institute of Life Sciense), was confirmed as previous- ly described [30].

\section{Light microscopy}

After washing the sections three times with PBS containing $0.005 \%$ saponin, the sections were incubated for $48 \mathrm{hr}$ with horseradish peroxidase-labelled sheep antimouse IgG antibody at a final dilution of $1: 40$. After washing with PBS containing $0.005 \%$ saponin, the sections were fixed again for $10 \mathrm{~min}$ in the fixative containing $1 \%$ glutaraldehyde in PBS. After washing with PBS, the sections were incubated for $30 \mathrm{~min}$ in $\mathrm{DAB}$ solution (50 mM Tris buffer containing 0.03\% 3,3/-diaminobenzidine tetrahydrochloride), and then incubated for $5 \mathrm{~min}$ in the DAB solution containing $0.01 \% \mathrm{H}_{2} \mathrm{O}_{2}$. The sections were embedded with $50 \%$ glycerine in PBS and examined by light microscopy.

\section{Electron microscopy}

Sections displaying typical immnoreactive features of sprouting at nodes of Ranvier were briefly washed in PBS and postfixed at $4{ }^{\circ} \mathrm{C}$ in $2 \% \mathrm{OsO}_{4}$ in the $0.1 \mathrm{M}$ sodium cacodylate buffer for $1 \mathrm{hr}$. After washing in PBS, the sections were then dehydrated in a graded series of ethanol, followed by QY-1, and embedded in Epon 812. Ultrathin sections were made on an LKB Ultrotome, and examined in a JEOL 100SX electron microscope without any electron staining. As a negative control, sections were incubated with non-immune mouse IgG instead of antisynaptotagmin $\mathrm{mAb}$, and processed in the same manner as above for light and electron microscopy.

\section{Results}

\section{Light microscopy}

In the injured nerve, the site of ligation was identified as a constricted portion in the sciatic nerve. Numerous thin strands with strong synaptotagmin immunoreactivity were demonstrated in the area up to approximately $2.0 \mathrm{~mm}$ proximal to the lesion (Fig. 1a). The immmnoreactive thin strands were seen extending along the surface of the myelin sheath. Strongly immunostained structures presumed to be the modified nodes of Ranvier in response to injury were also identified(Fig. 1b). The thin strands which emanated from such nodes of Ranvier and extended in both proximal and distal directions along the surface of the parent fibers possessed distinct synaptotagmin immunoreactivity.

The normal sciatic nerve immnostained with the antisynaptotagmin $\mathrm{mAb}$ appeared totally non-immunoreactive (data not shown).

\section{Electron microscopy}

Electron microscopic observations demonstrated that in the normal nerve fibers, the nodes of Ranvier exhibited no immunoreaction (Fig. 2) and that in the injured nerve fibers, the strongly immunostained structures seen by light microscopy were regenerating sprouts emanating from the 

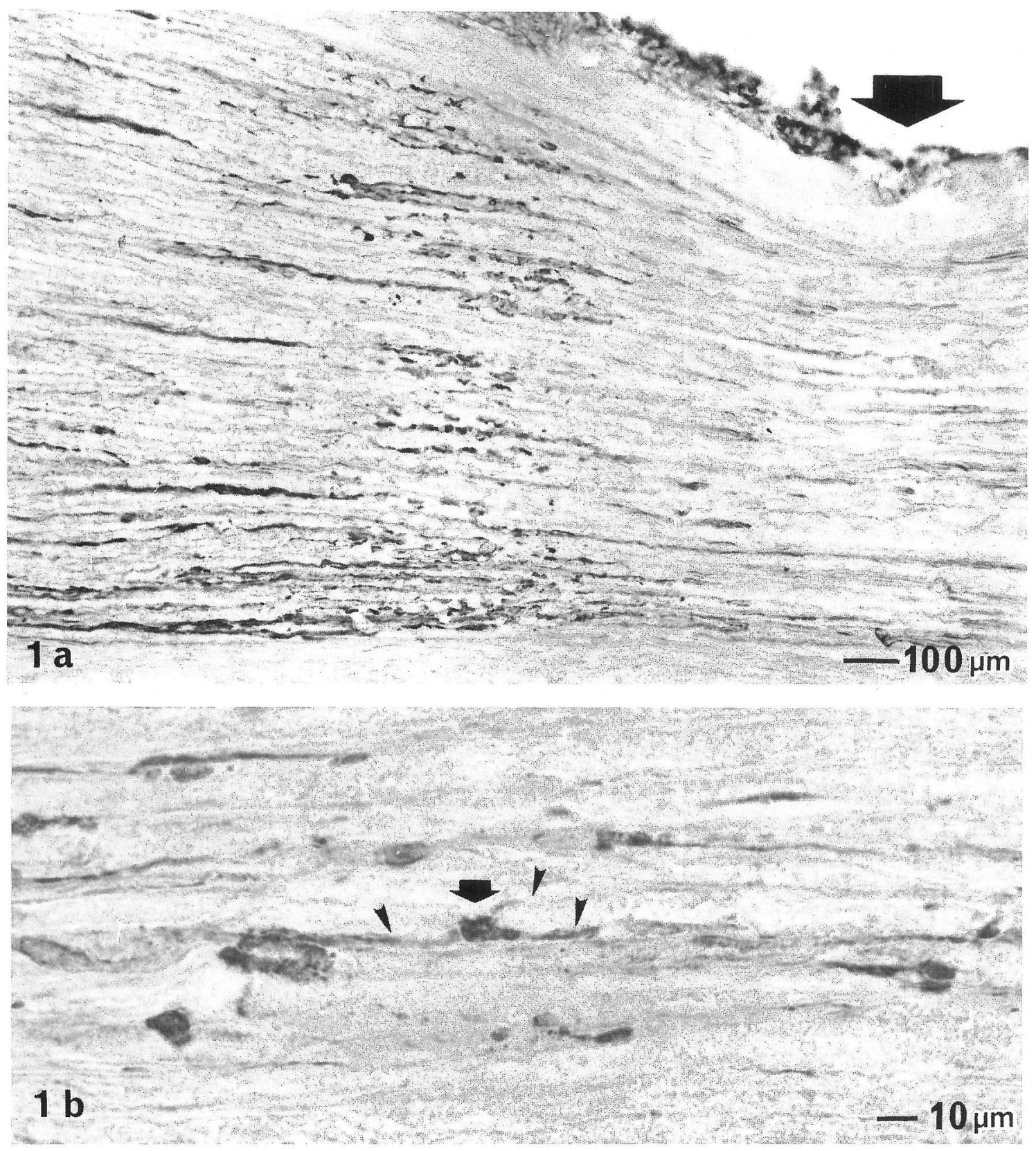

Fig. 1. Light micrographs showing longitudinal sections of the injured rat sciatic nerve immunostained with anti-synaptotagmin antibody. a: Within the region up to approximately $2.0 \mathrm{~mm}$ proximal to the lesion (large arrow), numerous immunoreactive thin strands are seen. b: At a higher magnification, a strongly immunostained structure presumed to be the modified node of Ranvier (large arrow) is seen. Thin strands are emanating from the node of Ranvier and extending in both proximal and distal directions along the surface of the parent fibers (arrow heads). Bars $=100 \mu \mathrm{m}$ in (a) and $10 \mu \mathrm{m}$ in (b).

nodes of Ranvier which were modified in response to injury.

The early sprouts were characterized by evagination of nodal axolemma as local small projections into the nodal gap, that contacted the Schwann cell basal lamina (Fig. 3). Similar axonal projections were also found at the paranodal regions. These sprouts contained small vesicles (ca, $50 \mathrm{~nm}$ in diameter), vacuoles $(100-200 \mathrm{~nm}$ ) and multivesicular bodies. They contained no cytoskeletal elements such as neurofilaments and microtubules. In these early sprouts, strong synaptotagmin immunoreactivity was primarily demonstrated on the small vesicles and vacuoles. Many small immunoreactive vesicles and vacuoles were also found in the nodal part of the parent axons. Clusters of the immunoreactive vesicles were often found in direct contact with or in close proximity to 


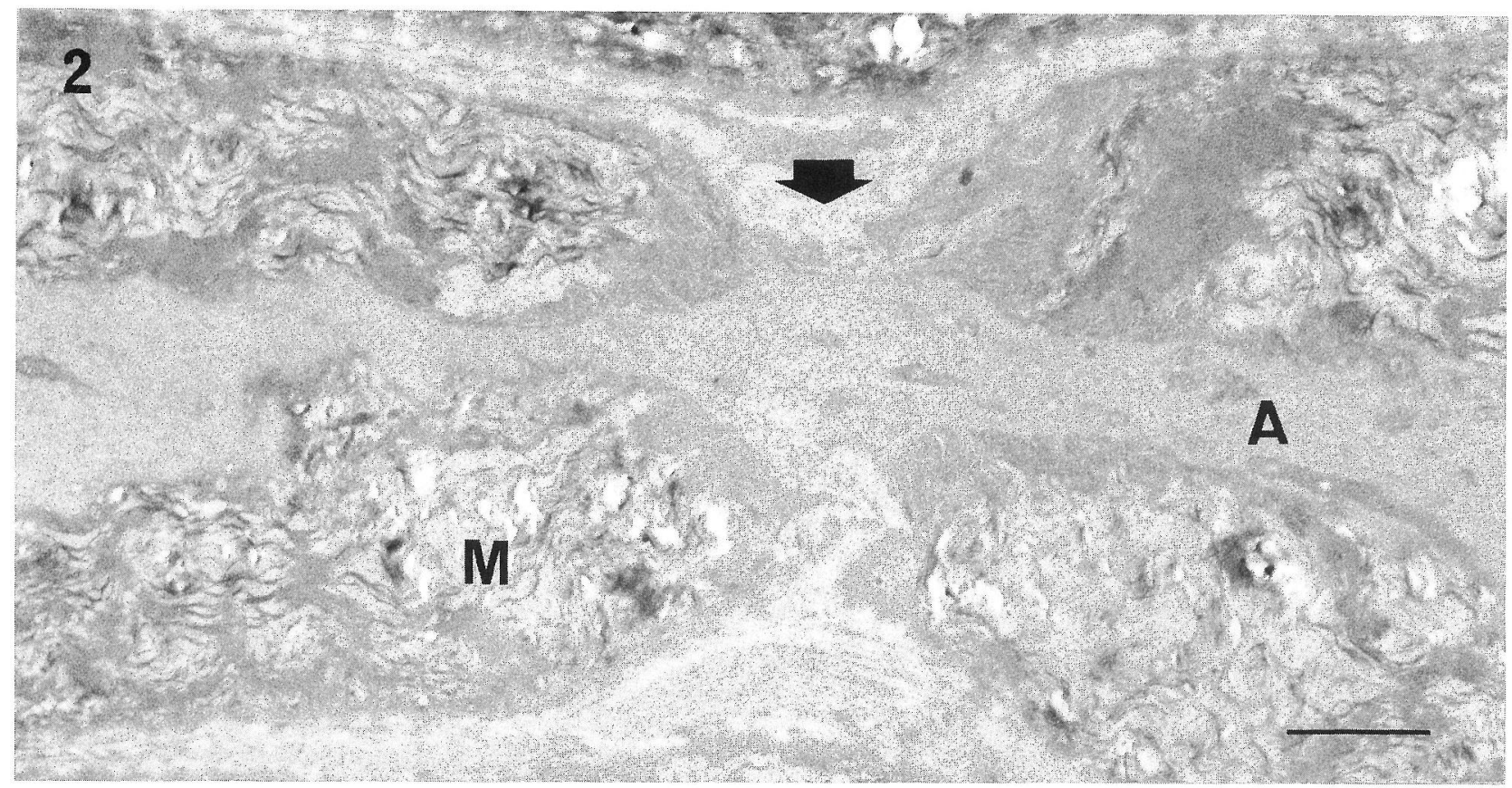

Fig. 2. Electron micrograph showing the normal node of Ranvier. No immunoreaction is recognized either on the nodal axolemma, vesicles or in the cytoplasm. A: axon, M: myelin sheath. Bar $=1 \mu \mathrm{m}$.

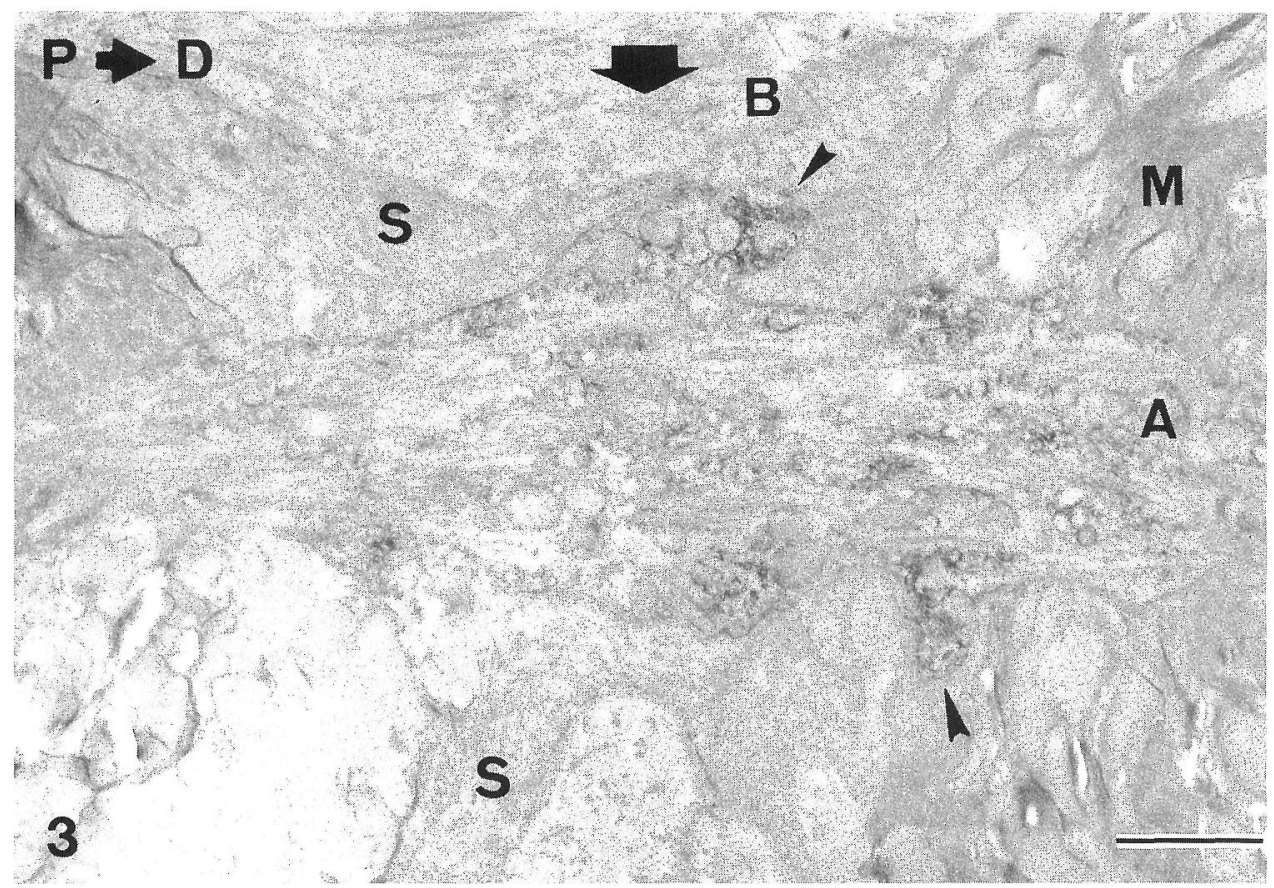

Fig. 3. Electron micrograph showing the node and paranode of the injured rat sciatic nerve. There are two sprouts (arrows) one of which emanates from the node(large arrow), while the other from the paranode. The nodal sprout contacts with the Schwann cell basal lamina. In the nodal part of the parent axons, many immunoreactive small vesicles and vacuoles are seen. Some of the immunoreactive vesicles are located in close proximity to the plasma membranes of the early sprouts and parent axon. A: the parent axon, $\mathrm{M}$ : myelin sheath. $\mathrm{P} \rightarrow \mathrm{D}:$ proximo-distal direction. Bar $=1 \mu \mathrm{m}$. 

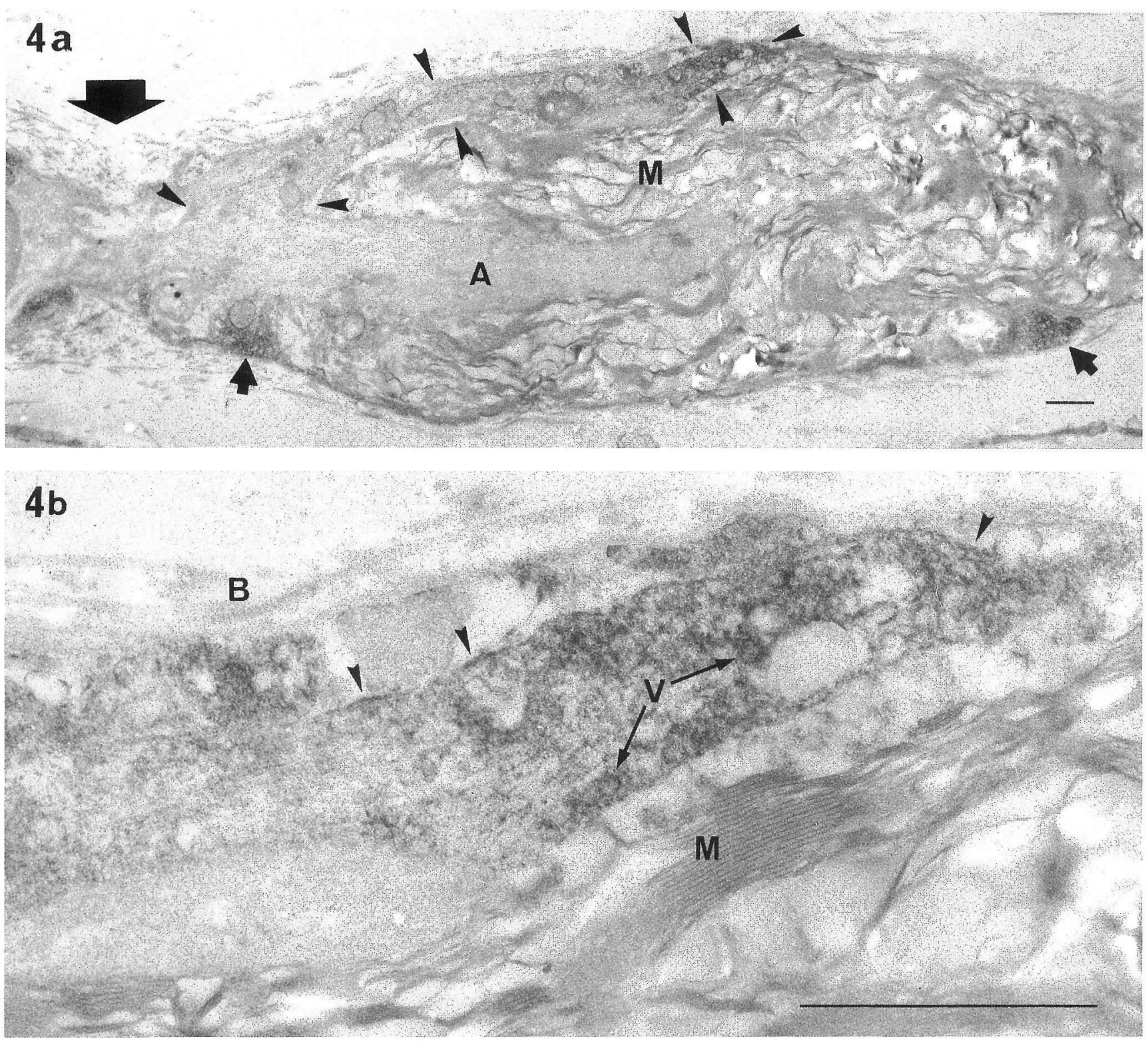

Fig. 4. Electron micrographs showing the longitudinal section of the well-developed regenerating sprout growing out from the node of Ranvier. a: The well-developed sprout (arrowheads) has grown out from the node of Ranvier (large arrow). Intense synaptotagmin immunoreactivity is recognized at the tip of the sprout, the growth cone. Whereas the plasma membranes of the stem region of the well-developed sprout which are continuous with those of the parent axon (A) are almost free of immunoreaction. Other two profiles of immunoreactive sprouts (arrows) are seen. M: myelin sheath. b: Higher magnification of the tip of the well-developed sprout seen in panel a. Intense synaptotagmin immunoreactivity is seen to be localized on the various kinds of vesicles (v) and plasma membranes(arrowheads) of the tip of the sprout, the growth cone, extending through the space between the Schwann cell basal lamina (B) and myelin sheath (M) of the parent fiber. Bar $=1 \mu \mathrm{m}$.

the plasma membranes of the early sprouts and parent axons. At these sites, the plasma membranes also appeared immunoreactive.

As the early sprouts extended through the space between the basal lamina and Schwann cell plasmalemma, they became well-developed sprouts (Fig. 4a). The tips of these sprouts always contained small vesicles, vacuoles and mitochondria, displaying the feature of typical growth cones. The stem regions of these sprouts where they were continuous with the parent axons contained an abundance of longitudinally oriented neurofilaments extending from the parent axon. In these well-developed sprouts, intense synaptotagmin immunoreactivity was demonstrated on the small vesicles, vacuoles and plasma membranes of their growth cones (Fig. 4b). The synaptotagmin immunoreactivity was distributed in association with the vesicles and vacuoles. Both focal and continuous staining patterns were noted on the plasma membranes of the growth 
cones. On the other hand, the stem regions of these sprouts exhibited almost no immunoreaction on the plasma membranes.

\section{Discussion}

The present study has demonstrated that synaptotagmin is located on the vesicles, vacuoles and plasma membranes of the early and well developed regenerating sprouts emanating from the nodes of Ranvier. In the well-developed sprouts, synaptotagmin is highly concentrated in the growth cones. These findings suggest that synaptotagmin-immunoreactive vesicles and vacuoles might be utilized for the supply of membrane components to the surface plasma membranes in the growth cones, and that synaptotagmin may participate in the regulation of the $\mathrm{Ca}^{2+}$-dependent membrane fusion between the vesicles and plasma membranes.

The role of synaptotagmin in the membrane fusion of synaptic vesicles with presynaptic plasma membranes in synapses has recently been investigated. Biochemically synaptotagmin binds $\mathrm{Ca}^{2+}$ with low affinity and forms a complex with synaptobrevin, SNAP25 and syntaxin, which are regarded as core components of membrane fusion machinery in synapses $[1,25]$. In the synaptotagmin knockout mice, $\mathrm{Ca}^{2+}$-dependent neurotransmitter release is severely impaired, whereas $\mathrm{Ca}^{2+}$-independent release remains normal [8]. These data suggest that synaptotagmin functions as $\mathrm{Ca}^{2+}$ sensor that triggers neurotransmitter release in synapses.

The growth cones in vitro contain various kinds of vesicles and vacuoles, which are presumed to be involved in the supply of membrane components to the surface plasma membranes $[4,5,29]$ and in the exocytosis of proteases [16]. The $\mathrm{Ca}^{2+}$-dependent membrane fusion in growth cones has been studied by the Phenninger's group, though proteins involved in the fusion have not been identified. They have developed a cell-free membrane expansion assay using intact growth cone particles and found that high potassium depolarization and calcium-ionophore treatment cause significant increases in exposed WGA binding sites within minutes $[22,23]$. By morphometric analysis, the source of the newly added plasma membranes has been identified as clear vesicles (150 $\mathrm{nm}$ in diameter).

In the regenerating sprouts emanating from the nodes of Ranvier in vivo, the source of the plasma membranes and the mode of the plasmalemmal expansion have not been clarified. In our previous study on the localization of synaptophysin, a synaptic vesicle-associated protein with four membrane-spanning domains, we have found that synaptophysin immunoreactivity is distributed throughout the cytoplasm, extending right up to the surface plasma membrane of the early regenerating sprouts emanating from the the nodes of Ranvier [21]. This finding suggests that surface plasma membrane of the early regenerating sprouts may be newly supplied by the fusion of synaptophysin-immunnoreactive vesicles with the axolemma.

In the present study, we have found that synaptotagmin-immunoreactive vesicles are distributed in the early regenerating sprouts and nodal portions of the parent axons. In the well-developed regenerating sprouts, we have further demonstrated that synaptotagminimmunoreactive plasma membranes are restricted to their tips, growth cones, which are filled with synaptotagminimmunoreactive vesicles and that the plasma membranes of the stem regions of the sprouts are almost free of synaptotagmin-immunoreactivity. This finding suggests that as the well-developed regenerating sprouts grow out further, synaptotagmin on the plasma membranes of the stem regions may be retrieved as endocytotic vesicles and selectively transported to the growth cones where synaptotagmin probably regulates membrane fusion.

\section{References}

1. Bennett, M. K., Calakos, N. and Scheller, R. H.: Syntaxin: a synaptic vesicle protein implicated in the docking of synaptic vesicles at presynaptic active zones. Science 257; 255-259, 1992.

2. Berthold, C.-H. and Rydmark, M.: Anatomy of the paranodenode-paranode region in the cat. Experientia 39; 964-976, 1983.

3. Berthold, C.-H. and Rydmark, M.: Electron microscopic serial section analysis of node of Ranvier in lumbosacral spinal roots of the cat: ultrastructural organization of nodal compartments in fibres of different sizes. J. Neurocytol. 12; 475-505, 1983.

4. Bray, D.: Model for membrane movements in the neural growth cone. Nature 244; 93-96, 1973.

5. Bunge, M. B.: Fine structure of nerve fibers and growth cones of isolated sympathetic neurons in culture. J. Cell Biol. 56; 713$735,1973$.

6. Cajal, S. R.: Degeneration and Regeneration of the Nervous Systems (facsimile of the 1928 edition), vol. 1, Hafner, London, 1968, pp, 141-166.

7. Debello, W. M., Betz, H. and Augustine, G. J.: Synaptotagmin and neurotransmitter release. Cell 74; 947-950, 1993.

8. Geppert, M., Goda, Y., Hammer, R. E., Li, C., Rosahl, T. W., Stevens, C. F. and Sudhof, T. C.: Synaptotagmin I: a major $\mathrm{Ca}^{2+}$-sensor for transmitter release at a central synapse. Cell 79; 717-727, 1994.

9. Haftek, J. and Thomas, P. K.: Electron-microscope observations on the effects of localized crush injuries on the connective tissues of peripheral nerve. J. Anat. 103; 233-243, 1968.

10. Hopkins, W. G. and Slack, J. R.: The sequential development of nodal sprouts in mouse muscles in response to nerve degeneration. J.Neurocytol. 10; 537-556, 1981.

11. Hopkins, W. G., Brown, M. C. and Keynes, R. J.: Nerve growth from nodes of Ranvier in inactive muscle. Brain Res. $222 ; 125-128,1981$.

12. Ichimura, T. and Ellisman, M. H.: Three-dimensional fine structure of cytoskeletal membrane interactions at nodes of Ranvier. J. Neurocytol. 20; 667-681, 1991.

13. Ide, C. and Kato, S.: Peripheral nerve regeneration. Neurosci. Res. Suppl., 13; 157-164, 1990.

14. Ide, C., Tohyama, K., Yokota, R., Nitatori, T. and Onodera, S.: Schwann cell basal lamina and nerve regeneration. Brain Res. 288; 61-75, 1983.

15. Ide, C., Miki, A., Tomatsuri, M., Okajima, S., Masutani, M., Kawakita, N., Mizoguchi, A., Arakawa, M. and Fujimoto, E.: Repairing process at the proximal stump of transected 
myelinated axons, and regenerating sprout formation at the node of Ranvier by ligation-injury in peripheral nerves. $A d v$. Neurotrauma Res. 3; 8-12, 1991.

16. Krystosek, A. and Seeds, N. W.: Plasminogen activator release at the neuronal growth cone. Science 213; 1532-1534, 1981.

17. Lanners, H. N. and Grafstein, B.: Early stages of axonal regeneration in the goldfish optic tract: an electron microscopic study. J. Neurocytol. 9; 733-751, 1980.

18. Matthew, W. D., Tsavaler,L. and Reichardt, L. F.: Identification of a synaptic vesicle-specific membrane protein with a wide distribution in neuronal and neurosecretory tissue. J. Cell Biol. 91; 257-269, 1981.

19. Maxwell, W. L., Irvine, A., Graham, D. I., Adams, J. H., Gennarelli, T. A., Tipperman, R. and Sturatis, M.: Focal axonal injury: the early axonal response to stretch. J. Neurocytol. 20; 157-164, 1991.

20. McQuarrie, I. G.: Effect of a conditioning lesion on axonal sprout formation of nodes of Ranvier. J. Comp. Neurol. 231; 239-249, 1985.

21. Okajima, S., Mizoguchi, A., Masutani, M., Tomatsuri, M., Tamai, K., Hirasawa, Y. and Ide, C.: Synaptotagmin immunocytochemistry in the regenerating sprouts from the nodes of Ranvier in injured rat sciatic nerve. Brain Res. 631; 133-136, 1993.

22. Pfenninger, K. H. and Maylie-Pfenninger, M.-F.: Lectin labeling of sprouting neurons II, Relative movement and appearance of glycoconjugates during plasmalemmal expansion. J. Cell Biol. 89; 547-559, 1981.

23. Pfenninger, K. H. and Pfenninger, M. M.: Lectin labeling of sprouting neurons I, regional distribution of surface glycoconjugates. J. Cell Biol. 89; 536-546, 1981.

24. Raine, C. S.: Differences between the nodes of Ranvier of large and small diameter fibres in the P. N.S.. J. Neurocytol. 11; 935-947, 1982.

25. Sollner, T., Whiteheart, S.W., Brunner, M., ErdjumentBromage, H., Geromanos, S., Tempst, P. and Rothman, J.E.: SNAP receptors implicated in vesicle targeting and fusion. Nature 362; 318-324, 1993.

26. Sudhof, T. C.: The synaptic vesicle cycle: a cascade of proteinprotein interactions. Nature 375; 645-653, 1995.

27. Tao-Cheng, J.-H. and Rosenbluth, J.: Development of nodal and paranodal membrane specializations in amphibian peripheral nerves. Dev. Brain Res. 3; 577-594, 1982.

28. Tomatsuri, M., Okajima, S. and Ide, C.: Sprout formation at the nodes of Ranvier of crush-injured peripheral nerves. Restor. Neurol. Neurosci. 5; 275-282, 1993.

29. Yamada, K. M., Spooner, B.S. and Wessells, N. K.: Ultrastructure and function of growth cones and axons of cultured nerve cells. J.Cell Biol. 49; 614-635, 1971.

30. Yoshida, A., Oho, C., Ohmori, A., Kuwahara, R., Ito, T. and Takahashi, M.: HPC-1 is associated with synaptotanmin and w-conotoxin receptor. J. Biol. Chem. 267; 24925-24928, 1992. 